\title{
$\widehat{A}$ Madridge \\ madridge Journal of Nursing \\ interconnecting Scientific World
}

Short Communication

Open Access

\section{Rehabilitation Concepts for the Acute Care Nurse}

\author{
Emily Havrilla* \\ Assistant Professor, Passan School of Nursing, Wilkes University, USA
}

\section{Article Info}

*Corresponding author:

Emily Havrilla

Assistant Professor

Passan School of Nursing

Wilkes University

84 W. South St.

Wilkes-Barre, PA 18766

USA

Tel: 570-408-4069

E-mail: emily.havrilla@wilkes.edu

Received: August 21, 2017

Accepted: September 20, 2017

Published: September 26, 2017

Citation: Havrilla E. Rehabilitation Concepts for the Acute Care Nurse. Madridge J Nurs. 2017; 2(2): 72-75.

doi: $10.18689 / \mathrm{mjn}-1000113$

Copyright: ( $\subset 2017$ The Author(s). This work is licensed under a Creative Commons Attribution 4.0 International License, which permits unrestricted use, distribution, and reproduction in any medium, provided the original work is properly cited.

Published by Madridge Publishers

\begin{abstract}
The integration of rehabilitation concepts into nursing practice across the continuum of care is crucial to the achievement of positive client outcomes. The Association of Rehabilitation Nurses recommends that rehabilitation nurses are present in all healthcare settings in which rehabilitation is provided. However, a rehabilitation nurse is not always available. Based upon the incorporation of rehabilitation concepts into educational programs, the acute care nurse should be able to recognize aspects of rehabilitation in his or her role. The aim of this article is to review competencies of rehabilitation nursing and discuss the role of the acute care nurse in the care of rehabilitation clients in the absence of a rehabilitation nurse.
\end{abstract}

Keywords: Rehabilitation nursing; Rehabilitation concepts; Rehabilitation nursing competency; Acute care nursing.

The concepts of rehabilitation nursing have historically been integrated into the role of nursing. Florence Nightingale, in the beginning of modern nursing, had a holistic perspective and included rehabilitation practices in her nursing care [1]. The inclusion of rehabilitation concepts in nursing practice and education is considered essential in contemporary nursing. In the Association of Rehabilitation Nurses (ARN) position statement "Inclusion of Rehabilitation Concepts in BSN Programs", the formal recommendation is made to incorporate rehabilitation concepts into baccalaureate programs [2]. This recommendation is based upon the historic use of rehabilitation techniques by nurses, the needs of individuals with disease or injury that impact function, and the holistic nature of rehabilitation nursing. Interprofessional care of patients and families, and the need for planning beyond acute care through case management, also underscore the need for the integration of rehabilitation nursing concepts in education.

Furthermore, there is an increasing need for rehabilitation services including rehabilitation nursing. The World Health Organization organized a meeting entitled "Rehabilitation 2030: A Call for Action" in which participants acknowledged an increasing demand for rehabilitation services. This reflects changing demographics of people living longer with chronic illness and injury [3]. The goals of rehabilitation ultimately include improved quality of life. However, familiarity with the primary goals of prevention of injury, maintenance of function, rehabilitation and restoration will help to frame rehabilitation needs.

\section{Rehabilitation Nursing}

Rehabilitation nursing is defined by the ARN as "the diagnosis and treatment of human responses of individuals and groups to actual and potential health problems related to impairments in function and lifestyle" [4]. The ARN advocates for the inclusion of rehabilitation nurses in all healthcare settings where rehabilitation is provided. Rehabilitation nurses have a holistic view of care and are able to take a comprehensive approach in education for clients and families faced with chronic illness or disability. Additionally, they have experience in forming partnerships and functioning as members of the interprofessional team [5]. 
ARN identified the need for a model to describe the competencies for the specialty of rehabilitation nursing. The model developed is congruent with the nursing paradigm of nurse, person, environment, and health. The Competency Model for Professional Rehabilitation Nursing speaks to the role of the rehabilitation nurse across four domains: nurse-led interventions; promotion of health and successful living; leadership; and interprofessional care. These domains reflect all aspects of the role of the rehabilitation nurse. Nurse-led interventions include the use of evidence-based practice and supportive technology to meet the needs of the client and provide family centered-care. Promotion of health and successful living describes the role of the nurse in promoting health and preventing further disability. The focus is on selfmanagement and independent living in the community. Leadership describes the role of the rehabilitation nurse as a care provider and team member. The nurse is knowledgeable and accountable and serves as an advocate for clients. Lastly, the nurse functions as an effective member of the rehabilitation team in the domain of interprofessional care. The model reflects the holistic nature of rehabilitation nursing practice [6]. Its design includes fourteen competencies described at three levels of nurse proficiency: beginner, intermediate, and advanced. ARN recommends that the Competency Model for Professional Rehabilitation Nursing be used as a foundation for the inclusion of rehabilitation concepts in curricula.

\section{Role of the Acute Care Nurse}

Despite the ARN recommendation for the inclusion of rehabilitation nurses in all settings where rehabilitation is provided [5], there are often settings where a certified rehabilitation registered nurse is not available. In these settings, the nurse has the responsibility of comprehensively addressing client and family needs. Nurses should be able to identify the rehabilitation aspects of their role based on the integration of these concepts in their educational programs [7].

There are numerous examples of the applicability of the concepts of rehabilitation nursing in the role of the acute care nurse. The examples may be grouped into three primary scenarios in the acute care setting: trauma or injury related; acute or chronic illness; and clients with a restorative focus.

\section{Trauma or Injury Scenario}

The acute care nurse provides care for clients who have experienced trauma or injury throughout the medical center. This may be at a point of access to the system such as the emergency department, or in inpatient areas including critical care and medical-surgical units. Tertiary care centers with a Level I Trauma Center designation usually are able to provide comprehensive care for patients including rehabilitation [8]. The nurse will be a member of the interprofessional team and participate in early interventions to prevent further injury and maintain function. In the case of a Level I Trauma Center, the rehabilitation interprofessional team members may be consulted to provide care recommendations and guidance. This may include an expert rehabilitation registered nurse. Rehabilitation team members may be specifically assigned to provide early interventions in critical care areas.

Trauma clients may progress through the acute care continuum until medically stable and appropriate for transfer to an acute rehabilitation hospital or other setting. At each level of care, the primary acute care nurse will be responsible for the integration of rehabilitation interventions into the plan of care.

\section{Acute or Chronic Illness Scenario}

Often clients with an acute illness or exacerbation of a chronic illness are admitted to a medical-surgical area. In addition to nursing interventions directed towards the primary diagnosis, the nurse will need to include interventions in the plan of care to address alterations in self-management and independent living. Early consultation with rehabilitation members of the interprofessional team to address altered functional capabilities related to the illness will facilitate successful discharge planning and care transition. Likewise, clients admitted with a secondary diagnosis that impacts functional independence or self-management will necessitate an evaluation of the continuance of interventions already in place.

\section{Restorative Care Scenario}

Admission of clients to acute care from long-term care, personal care, and other similar settings will require the nurse to evaluate the restorative care plan. Restorative nursing care is focused on the restoration or maintenance of function. It is designed to facilitate independence in activities of daily living in the presence of physiologic changes. The maintenance of functional routines will facilitate the preservation of functional abilities in hospitalized clients and provide for improved outcomes.

\section{Key Aspects of Rehabilitation}

The acute care nurse will implement rehabilitation aspects of care as related to the client, family, team, and transitional plan. Client interventions will be based upon assessment of individual needs, the prevention of injury, and maintenance of function. Client education will be provided within the knowledge level of the nurse and may include recommendations from the rehabilitation team. As an advocate, the nurse will need to include advocacy for client rehabilitation needs and self-management. Implementation of nursing care that includes a rehabilitation focus will ultimately improve quality of life.

Family and care-giver support is crucial for successful client outcomes in all healthcare settings. The family requires support, education, assistance with planning, and the identification of resources for independent living or continued rehabilitation. These are essential not only for the care giver, 
but also for the achievement of successful outcomes for the individual client. Support provided by the nurse includes the unique ability to identify and discuss changes to family roles, the impact of altered functional level, and the provision of emotional support. This may include referral to formal sources of support. The nurse's role includes family education regarding the specifics of the care to be provided and the issues that the client will experience. The nurse may coordinate or participate in planning for transfer across the healthcare continuum. Nursing expertise is included in a realistic assessment of the care needs of the client and the expectations of care givers. A disconnect between client and family expectations and the actual needs of the client may lead to an unsuccessful transition. Coordination with case management will facilitate linkage to resources and the development of an appropriate life plan as well as transition to acute or subacute rehabilitation settings.

In the inpatient setting, the acute care nurse will continue to be an essential member of the interprofessional team; however, rehabilitation needs should be addressed by consultation with the rehabilitation team or inclusion of appropriate rehabilitation professionals as team members. The rehabilitation team members may ask the acute care nurse to participate in the implementation of interventions to achieve rehabilitation goals. This may include strategies for increased mobility and independence with activities of daily living by occupational therapy, specific transfer techniques and instructions regarding the use of assistive devices by physical therapy, and swallowing techniques to prevent aspiration in clients with dysphagia by speech therapy. Interprofessional assessment and discussion to develop a client plan of care will best serve to meet the client's needs.

\section{Rehabilitation Competencies}

The utilization of the Competency Model for Professional Rehabilitation Nursing as a framework for the implementation of rehabilitation concepts into academic curricula facilitates the translation of the model to support recommendations for the inclusion of rehabilitation concepts in practice by the acute care nurse. The rehabilitation nursing competencies [6], are able to be mapped to examples of rehabilitation aspects of the role of the acute care nurse. Tables 1 through 4 provide examples of the rehabilitation competencies in each domain, as articulated by ARN [6], with associated practice examples for the acute care nurse. The acute care nurse may use these examples to identify concepts of rehabilitation nursing that are present within his or her role.
Table 1. Domain 1 - Nurse-led Interventions and Acute Care Nurse Activities

\begin{tabular}{|c|c|}
\hline Rehabilitation Competency & Acute Care Nurse Activities \\
\hline $\begin{array}{l}\text { Use Supportive Technology to } \\
\text { Improve Quality of Life }\end{array}$ & $\begin{array}{l}\text { - Assists client in utilizing previously } \\
\text { implemented supportive technology } \\
\text { when hospitalized. } \\
\text { - Collaborates with interprofessional team } \\
\text { in identifying alterations in function and } \\
\text { need for supportive technology. }\end{array}$ \\
\hline $\begin{array}{l}\text { Implement Nursing \& } \\
\text { Interprofessional Interventions } \\
\text { Based on Evidence }\end{array}$ & $\begin{array}{l}\text { - Uses nursing specific evidence-based } \\
\text { protocols at level of expertise. } \\
\text { - Implements interprofessional and/or } \\
\text { rehabilitation team recommendations } \\
\text { for protocols. }\end{array}$ \\
\hline $\begin{array}{l}\text { Provide Client and Caregiver } \\
\text { Education in Relation to } \\
\text { Disability, Chronic Illness, \& } \\
\text { Health Management }\end{array}$ & $\begin{array}{l}\text { - Assesses client and caregiver learning } \\
\text { needs including assessment related to } \\
\text { rehabilitation needs. } \\
\text { - Provides client and caregiver education } \\
\text { at level of expertise. } \\
\text { - Consults with rehabilitation team to } \\
\text { provide education as appropriate. } \\
\text { - Reinforces education with clients. }\end{array}$ \\
\hline
\end{tabular}

Table 2. Domain 2 - Promotion of Health and Successful Living and Acute Care Nurse Activities

\begin{tabular}{|l|l|}
\hline Rehabilitation Competency & Acute Care Nurse Activities \\
\hline $\begin{array}{l}\text { Promote Health \& Prevent } \\
\text { Disability }\end{array}$ & $\begin{array}{l}\text { - Includes a rehabilitation focus in risk } \\
\text { assessment and goal setting for health } \\
\text { promotion and risk prevention. }\end{array}$ \\
\hline Foster Self-management & $\begin{array}{l}\text { Incorporates client knowledge and } \\
\text { readiness to learn in nursing assessment } \\
\text { related to illness or disability. } \\
\text { - Includes evaluation of self-management. } \\
\text { - Collaborates with the interprofessional } \\
\text { team in the area of self-management. }\end{array}$ \\
\hline $\begin{array}{l}\text { Promote \& Facilitate Safe and } \\
\text { Effective Care Transitions }\end{array}$ & $\begin{array}{l}\text { The acute care nurse participates in } \\
\text { planning and facilitating care transitions. } \\
\text { Care transitions that include complex } \\
\text { planning related to rehabilitation needs } \\
\text { may require consultation with expert. }\end{array}$ \\
\hline
\end{tabular}

Table 3. Domain 3 - Leadership and Acute Care Nurse Activities

\begin{tabular}{|l|l|}
\hline Rehabilitation Competency & Acute Care Nurse Activities \\
\hline Promote Accountability for Care & $\begin{array}{l}\text { Provides safe, ethical care. } \\
\text { - Participates in quality improvement plan } \\
\text { in healthcare agency. }\end{array}$ \\
\hline - Includes rehabilitation concepts in care.
\end{tabular}


Table 4. Domain 4 - Interprofessional Care and Acute Care Nurse Activities

\begin{tabular}{|c|c|}
\hline Rehabilitation Competency & Acute Care Nurse Activities \\
\hline $\begin{array}{l}\text { Develop Interprofessional } \\
\text { Relationships }\end{array}$ & $\begin{array}{l}\text { - Serves as a member of the } \\
\text { interprofessional team. } \\
\text { - Recommends inclusion of rehabilitation } \\
\text { professionals as members of the } \\
\text { interprofessional team. }\end{array}$ \\
\hline $\begin{array}{l}\text { Implement an Interprofessional } \\
\text { Holistic Plan of Care }\end{array}$ & $\begin{array}{l}\text { - Identifies problems, develops joint goals } \\
\text { with patient } \\
\text { - Seeks rehabilitation expertise in } \\
\text { formulating interprofessional plan of } \\
\text { care. } \\
\text { - Evaluates effectiveness of nursing } \\
\text { interventions }\end{array}$ \\
\hline $\begin{array}{l}\text { Foster Effective Interprofessional } \\
\text { Collaboration }\end{array}$ & $\begin{array}{l}\text { - Serves as a nursing member of the } \\
\text { interprofessional team. } \\
\text { - Recommends inclusion of rehabilitation } \\
\text { concepts and goals in plan of care. } \\
\text { - Fosters positive team interactions. }\end{array}$ \\
\hline
\end{tabular}

\section{Conclusion}

A review of the competencies of rehabilitation nursing and a mapping of the competencies to acute care interventions demonstrate the rehabilitation concepts inherent in nursing care. Awareness of the rehabilitation concepts associated with many care interventions will facilitate effective care planning for clients with rehabilitation needs by the acute care nurse. The nurse will be able to serve as an advocate for the acute care client in meeting rehabilitation needs and articulate goals for planning with the inclusion of the client and family. It is recommended that rehabilitation concepts continue to be integrated into nursing education. Continuing education for the acute care nurse should include the application of concepts of rehabilitation.

\section{Conflict of Interest Statement}

The author declares that there is no conflict of interest.

\section{Reference}

1. Dossey BM. Holistic nursing: from Florence Nightingale's historical legacy

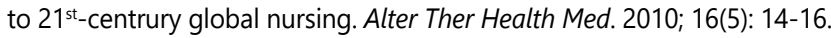

2. Association of Rehabilitation Nurses. Inclusion of rehabilitation concepts in BSN programs: an ARN position statement. The Association of Rehabilitation Nurses Website. August 17, 2017.

3. World Health Organization. Rehabilitation 2030: a call for action. Meeting report of Rehabilitation 2030: A Call for Action; 2017 Feb 6-7; Geneva, Switzerland: World Health Organization; 2017.

4. Association of Rehabilitation Nurses. Standards and Scope of Rehabilitation Nursing Practice $6^{\text {th }}$ ed. Chicago, IL: Association of Rehabilitation Nurses; 2014.

5. Association of Rehabilitation Nurses. Position statement: the appropriate inclusion of rehabilitation nurses where rehabilitation is provided. The Association of Rehabilitation Nurses Website. August 17, 2017.

6. Vaughn $\mathrm{S}$, Mauk, $\mathrm{KL}$, Jacelon $\mathrm{CS}$. et al. The competency model for professional rehabilitation nursing. Rehabil Nurs. 2016; 41(1): 33-44. doi: 10.1002/rnj.225

7. Hawkey B, Williams J. Rehabilitation: the nurse's role. Orthop Nurs. 2001 5: 81-88. doi: 10.1054/joon.2001.0155

8. American Trauma Society. Trauma levels explained. August 19, 2017 